\title{
SUBLAYER EFFECT NEAR THE WALL IN THE PRESENCE OF SVEDOV - BINGHAM FLOW
}

\author{
NGUYEN HUU CHI \& PHAM HOAI THANH \\ Hanoi Technology University
}

\begin{abstract}
SUMMARY. In this paper we study the stationary flow of viscous - plastic fluid in the horizontal cylindrical tube (Svedov Bingham's flow) with the assumption of existence of the viscous adherent sublayer near the wall. The obtained results are estimated and compared with those of Svedov Bingham flow. We also inspect the "near the wall effect" which was showed in the work of Smoldurey \& Xaponov and have some notes and estimation about it.
\end{abstract}

\section{§. INTRODUCTION}

The viscous - plastic liquid model (Svedov - Bingham's model) is used in some problems on oil production or fluid - solid mixture transport in pressure tube $[2,3,4]$. Many problems on stationary flow of viscous - plastic weve solved and showed in literatures.

The problem on laminar stationary cylindrical tube flow of viscous - plastic mixture with viscous non - adherent sublayer near the wall has solved in [4]. Besides, it is known that all the fluids in sluries (fluid - solid mixtures) have adhesion in shear. In this paper we have studied the stationary laminar pressure flow of viscous - plastic fluid in the horizontal cylindrical tube with the assumption of existence of viscous - adherent sublayer with small thickness $\delta$ and viscosity $\eta_{0}$ near the wall.

The obtained system of correspondent motion equations with its boundary conditions has been solved completely. The velocity profile and discharge of considered flow were compared with those of Svedov - Bingham flow. We also have some estimations and compare with results showed in [4].

\section{§2. SYSTEM OF MOTION EQUATIONS AND ITS SOLUTION}

Consider the one - dimensional stationary laminar pressure flow of viscous - plastic fluid in the horizontal cylindrical tube generated by constant pressure gradient with the assumption of existence of the viscous adherent sublayer near the wall, i.e. there is the sublayer of viscous fluid with the very small thickness which was formed and moves near the wall. Here we don't care about a cause of formation of sublayer. The picture of such flow can be observed in the tube transport of fluid - solid mixtures.

Denote by $R$ a radius of the tube, $R_{1}=R-\delta$ a radius of shared boundary between the main flow and the sublayer, $\delta$ a thickness of sublayer and $\operatorname{Or} \theta z$ a system of cylindrical coordinates having $O z$ coincide with axis of the cylinder (Fig. 1).

It is easy to find from continuity equations (for the main flow of viscous - plastic fluid and the flow of viscous fluid in sublayer) and symmetry of flow that the flow characteristics depend only on the radial coordinate $r$. 


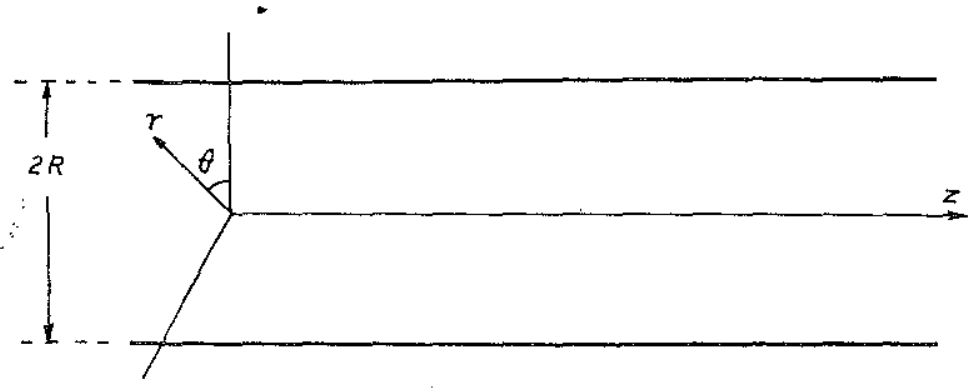

Fig. 1. The tube and system of coordinates

From Navier - Stokes' equations, Henki - Iliusin equations, the equations of continuty and symmetry of flow wa obtain the following motion equations:

$$
\begin{array}{ll}
\frac{d^{2} u_{s u b}}{d r^{2}}+\frac{1}{r} \frac{d u_{s u b}}{d r}+\frac{\Delta p}{\ell \eta_{0}}=0, & R_{1} \leq r \leq R \\
\frac{d^{2} u}{d r^{2}}+\frac{1}{r} \frac{d u}{d r}+\frac{\Delta p}{\ell \eta}-\frac{\tau_{0}}{\eta r}=0, & r_{0} \leq r \leq R_{1}
\end{array}
$$

with boundary conditions:

$$
\begin{aligned}
& \left.u_{s u b}\right|_{r=R}=0 \\
& \left.u_{s u b}\right|_{r=R_{1}}=\left.u\right|_{r=R_{1}} \\
& -\left.\eta_{0} \frac{d u_{s u b}}{d r}\right|_{r=R_{1}}=\tau_{0}-\left.\eta \frac{d u}{d r}\right|_{r=R_{1}} \\
& \left.\frac{d u}{d r}\right|_{r=r_{0}}=0
\end{aligned}
$$

where $u_{s u b}$ - the velocity of flow in the viscous sublayer

$u$ - the velocity of flow in viscou - plastic region

$\eta$ - the structural viscousity of viscou - plastic fluid

$r_{0}$ - the radius of elastic core

$\frac{\Delta p}{\ell}$ - the pressure gradient

$\tau_{0}$ - the ultimate shear stress (yield stress).

From balance condition of elastic core we have

$$
\tau_{0}=\frac{\Delta p}{2 \ell} r_{0}
$$

Integrating (2.1), (2.2) and by using the equality (2.7) we have the solution of the equation system $(2.1),(2.2)$ satisfying the boundary conditions (2.3) $-(2.6)$ as follows:

$$
\begin{aligned}
& u_{s u b}=\frac{\Delta p}{4 \ell \eta_{0}}\left(R^{2}-r^{2}\right) ; \quad R_{1} \leq r \leq R \\
& u=\frac{\Delta p}{4 \ell \eta}\left(R_{1}^{2}-r^{2}\right)-\frac{\tau_{0}}{\eta}\left(R_{1}-r\right)+\frac{\Delta p}{4 \ell \eta_{0}}\left(R^{2}-R_{1}^{2}\right) ; \quad r_{0} \leq r \leq R_{1}
\end{aligned}
$$

The velocity in the elastic core $u_{0}$ is determined by condition

$$
u_{0}=\left.u\right|_{r=r_{0}}, \quad r \leq r_{0}
$$


we obtain

$$
u_{0}=\frac{\Delta p}{4 \ell \eta}\left(R_{1}^{2}-r_{0}^{2}\right)-\frac{\tau_{0}}{\eta}\left(R_{1}-r_{0}\right)+\frac{\Delta p}{4 \ell \eta_{0}}\left(R^{2}-R_{1}^{2}\right) ; \quad r \leq r_{0}
$$

using equality (2.7) yields

$$
u_{0}=\frac{\Delta p}{4 \ell \eta}\left(R-r_{0}\right)^{2}+\frac{\Delta p}{4 \ell \eta_{0}}\left(R^{2}-R_{1}^{2}\right) ; \quad r \leq r_{0}
$$

The formulae (2.8), (2.9) and (2.11) (or (2.12)) represent the velocity profile in the cross section of the tube.

It should be noted that, at the shared boundary between the main flow and the sublayer near the wall $r=R_{1}$, the conditions (2.4) and (2.5) are satisfied but the condition of "smoothness" of velocity profill is not, i.e.

$$
\left.\frac{d u_{* u b}}{d r}\right|_{r=R_{1}} \neq\left.\frac{d u}{d r}\right|_{r=R_{1}}
$$

However, at this shared boundary, the inside shear stress is equal to the outside one as we showed in the condition (2.5) above.

Fig. 2 shows a velocity distribution in the cross - section of the tube.

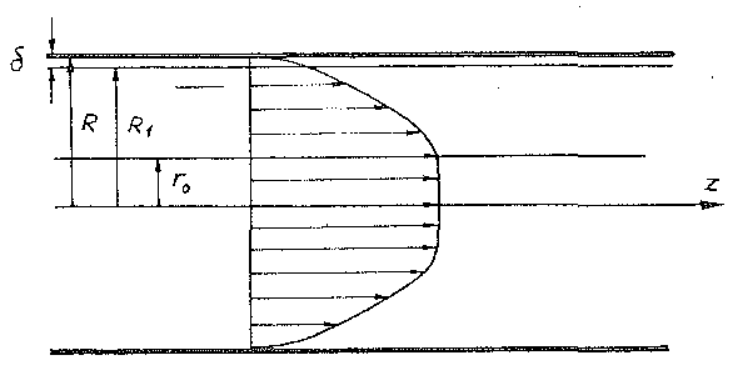

Fig. 2. Velocity profile in the cross-section

It is well-known the velocities of Svedov - Bingham flow are determined by the following formulae $[2,4]$ :

$$
\begin{gathered}
u_{v}=\frac{\Delta p}{4 \ell \eta}\left(R^{2}-r^{2}\right)-\frac{\tau_{0}}{\eta}(R-r) ; \quad \tau_{0} \leq r \leq R \\
u_{0 v}=\frac{\Delta p}{4 \ell \eta}\left(R-r_{0}\right)^{2} ; \quad r \leq r_{0}
\end{gathered}
$$

and its discharge is

$$
Q_{v}=\frac{\pi \Delta p}{8 \ell \eta}\left(R^{4}-\frac{4}{3} R^{3} r_{0}+\frac{1}{3} r_{0}^{4}\right)
$$

Besides, from (2.8) we have

$$
\left.u_{s u b}\right|_{r=R_{1}}=\frac{\Delta p}{4 \ell \eta_{0}}\left(R^{2}-R_{1}^{2}\right)
$$

Comparing (2.9), (2.12) and (2.13), (2.14) we find that with the assumption of existence of viscous adherent sublayer near the wall (thickness $\delta=R-R_{1}$ ), the flow of viscous - plastic fluid in the tube is similar to the flow of viscous - plastic fluid in the tube with radius $R_{1}$ while this flow slides inside the viscous sublayer with velocity equal to

$$
\left.u_{s u b}\right|_{r=R_{1}}=\frac{\Delta p}{4 \ell \eta_{0}}\left(R^{2}-R_{1}^{2}\right)
$$


Moreover, the solution (2.8) shows that, the velocity distribution in the viscous sublayer $\leq r \leq R$ ) is same similar to the velocity distribution of Poiselle flow, i.e. it looks like a part $\leq r \leq R$ ) of stationary flow of viscous fluid in the whole of the tube.

The discharge of considered flow is

$$
\begin{aligned}
Q & =\pi r_{0}^{2} u_{0}+2 \pi \int_{r_{0}}^{R_{1}} u r d r+2 \pi \int_{R_{1}}^{R} u_{s u b} r d r= \\
& =\frac{\pi \Delta p}{8 \ln }\left(R_{1}^{4}-\frac{4}{3} R_{1}^{3} r_{0}+\frac{1}{3} r_{0}^{4}\right)+\frac{\pi \Delta p}{8 \ln \eta_{0}}\left(R^{4}-R_{1}^{4}\right) .
\end{aligned}
$$

\section{§3. DISCUSSION}

For the evaluation of the obtained results, we consider the differences of velocities in both es: the flow with and without the viscous adherent sublayer near the wall.

a) In the sublayer near the wall $R_{1} \leq r \leq R$, from the formulae (2.8) and (2.13) we have:

$$
\begin{aligned}
\Delta u_{9 u b} & =\frac{\Delta p}{4 \ell \eta_{0}}\left(R^{2}-r^{2}\right)-\frac{\Delta p}{4 \ell \eta}\left(R^{2}-r^{2}\right)+\frac{\tau_{0}}{\eta}(R-r)= \\
& =\frac{\Delta p}{4 \ell}\left(R^{2}-r^{2}\right)\left(\frac{1}{\eta_{0}}-\frac{1}{\eta}\right)+\frac{\tau_{0}}{\eta}(R-r) .
\end{aligned}
$$

b) In the viscous - plastic layer $r_{0} \leq r \leq R_{1}$, from the formulae (2.9) and (2.13) we have:

$$
\begin{aligned}
\Delta u & =\frac{\Delta p}{4 \ell \eta}\left(R_{1}^{2}-r^{2}\right)-\frac{\tau_{0}}{\eta}\left(R_{1}-r\right)+\frac{\Delta p}{4 \ell \eta_{0}}\left(R^{2}-R_{1}^{2}\right)-\frac{\Delta p}{4 \ell \eta}\left(R^{2}-r^{2}\right)+\frac{\tau_{0}}{\eta}\left(R-r_{0}\right)= \\
& =\frac{\Delta p}{4 \ell}\left(R^{2}-R_{1}^{2}\right)\left(\frac{1}{\eta_{0}}-\frac{1}{\eta}\right)+\frac{\tau_{0}}{\eta}\left(R-R_{1}\right) .
\end{aligned}
$$

c) In the elastic core $r \leq r_{0}$, from the formulae (2.12) and (2.14) we have:

$$
\Delta u_{0}=\frac{\Delta p}{4 \ell}\left(R^{2}-R_{1}^{2}\right)\left(\frac{1}{\eta_{0}}-\frac{1}{\eta}\right)+\frac{\tau_{0}}{\eta}\left(R-R_{1}\right) .
$$

According to Smondurev [4], the viscosity coefficient in sublayer near the wall $\eta_{0}$ has only merical value of structural viscosity coefficient in $\left(\frac{1}{10} \div \frac{1}{2}\right)^{*}$ so $\Delta u_{s u b} \geq 0$ for $R_{1} \leq r \leq R(=0$ the wall) and $\Delta u=\Delta u_{0}=\left.\Delta u_{s u b}\right|_{r=R_{1}}=$ const $>0$ for $r \leq R_{1}$.

For convenience of comparison, the velocity profiles in both the cases are expressed in Fig. 3

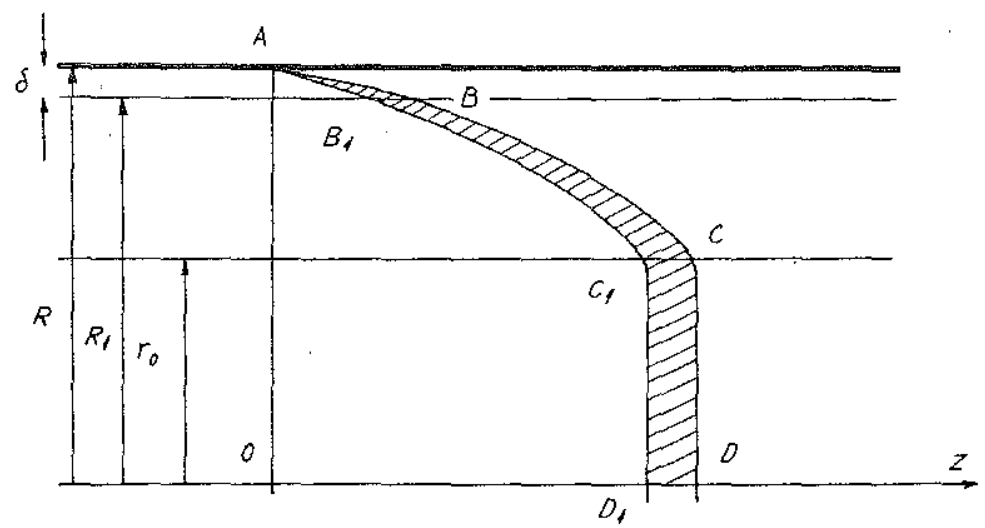

Fig. 3. Expression of radial longitudinal semisection and velocity profiles 
In Fig. $3 \mathrm{OAB}_{1} \mathrm{C}_{1} \mathrm{D}_{1}$ is the velocity profile of Svedov - Bingham flow (formulae (2.13), (2.14)). $\mathrm{OABCD}$ is the velocity profile of flow with sublayer near wall (formulae (2.8), (2.9), (2.11)). From $(2.15),(2.17)$ and $\left(^{*}\right)$ we have

$$
\Delta Q=Q-Q_{v}=\frac{\pi \Delta p}{8 \ell}\left(R^{4}-R_{1}^{4}\right)\left(\frac{1}{\eta_{0}}-\frac{1}{\eta}\right)+\frac{\pi \Delta p}{6 \ell \eta} r_{0}\left(R^{3}-R_{1}^{3}\right)>0
$$

and $\triangle Q$ is exactly the volume of rotatory body given by the complete revolution of the area $\mathrm{D}_{1} \mathrm{C}_{1} \mathrm{~B}_{1} \mathrm{ABCD}$ (the shaded region) around the tube axis $O Z$. Because of smallness of $\delta$ we can omit the terms containing high - power of $\delta$ in (3.4) and relative difference of discharges can be determined approximately by the formula

$$
\frac{\Delta Q}{Q_{v}} \simeq \frac{4 \delta\left(\frac{\eta}{\eta_{0}}-1+\frac{r_{0}}{R}\right)}{R\left[1-\frac{4 r_{0}}{3 R}+\frac{1}{3}\left(\frac{r_{0}}{R}\right)^{4}\right]}
$$

According to the $[4]$ in "near the wall effect", the boundary condition at $r=R_{1}=R-\delta$ was

$$
u_{\delta}=\frac{\Delta p}{4 \ell \eta_{0}}\left[R^{2}-(R-\delta)^{2}\right]
$$

and the distribution of slurry velocities in the tube with "near the wall effect" was expressed in Fig. 4 (Fig. 17, p. $52[4]$ )

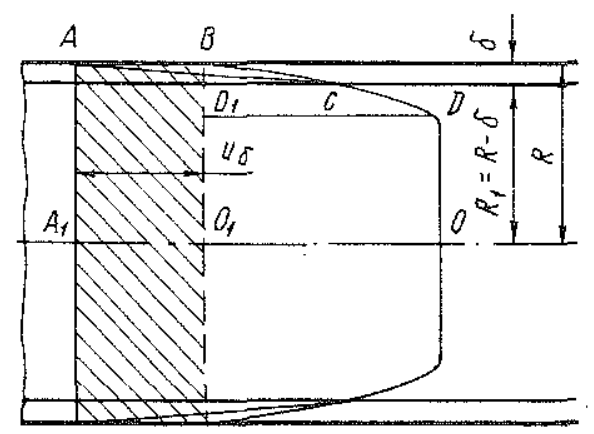

Fig. 4

The author wrote that the difference between discharges of this flow and Svedov - Bingham's one is the volume of rotatory body given by revolution of the shaded region in Fig. 4. Besides, the author determined the discharge of flow as follows

$$
\begin{aligned}
Q & =\pi r_{0}^{2}+2 \pi \int_{r_{0}}^{R_{1}} u r d r+2 \pi \int_{R_{1}}^{R} u_{\delta} r d r= \\
& =\frac{\pi \Delta p R_{1}^{4}}{8 \ell \eta}\left[1-\frac{4}{3}\left(\frac{\Delta p_{0}}{\Delta p}\right)+\frac{1}{3}\left(\frac{\Delta p_{0}}{\Delta p}\right)^{4}\right]+\frac{\pi \Delta p}{4 \ell \eta_{0}}\left(\frac{3}{2} R^{4}-R^{2} R_{1}^{2}+\frac{1}{2} R_{1}^{4}\right)
\end{aligned}
$$

(the formulae (II.25), p.52 and (II.26), p.53 [4]).

The explanations and formulae in this work are not so clear; moreover, the discharge determined is to large if we compare it with that of flow having viscous non-adherent (or adherent) sublayer near the wall. 


\section{$\S 4$. CONCLUSION}

The system of correspondent motion equations of considered flow had been solved completely. 'he obtained results show that:

The velocity distribution in viscous sublayer is similar to that of Poisell flow (for $R_{1} \leq r \leq R$ ) nd in the rest of considered flow it is similar to that of Svedov - Bingham's one while the latter ides inside viscous sublayer.

The additional discharge generated by sublayer effect is determined by the formular (3.4).

The results, which were showed in [4] ("near the wall effect") are not correct and especially le determined discharge by the formula (3.7) is too large

\section{REFERENCES}

Altsul A. D. Hydraulics and aerodynamics. Moscow, "Stroizdat", 1975 (in Russian).

Mirzadzanzade A. Kh. Problems of Hydrodynamics of viscous - plastic and viscous fluids in oil production. Pub. "Azer. Repub. Pub. of oil and science - tech. literatures", Baku, 1959 (in Russian).

Mirzadzanzade A. Kh., Entov B. M. Hydrodynamics in drilling. Moscow, "Nedra", 1985 (in Russian).

- Smoldurev A. E., Xafonov Iu. K. Pipe-line transport of concentrated slurries. Moscow, "Masinostroenie", 1973 (in Russian).

Faizullaev D. F. Hydrodynamics of one-and multiphase media. Taskent, "FAN" 1986 (in Russian).

Received March 13, 1994

\section{HIÊU ỨNG LỚP MỎNG SÁT THÀNH TRONG DÒNG CHẢY SVEDOV - BINGHAM}

Trong bài này chúng tôi xét dòng chảy dừng của chất lóng nhớt - déo trong ống trụ tròn nằm ;ang (dòng Svedov - Bingham) với gia thuyết là có tồn tại một lớp móng nhớt dính sát thành n. Kết quả thu được đã được so sánh với kết quả khi xét dòng Svedov - Bingham. Chúng tôi ng có vài chú ý và đánh giá khi xem xét "hiệu ứng sát thành" trình bày trong công trình của noldurev \& Xaforov. 